\title{
Packet Scheduling in Wireless Networks with Unreliable Links
} Jun $X \mathrm{u}^{1, \mathrm{a}}$, Jianfeng Yang ${ }^{1, \mathrm{~b}^{\star}}, \underset{\mathrm{Xie}^{1, \mathrm{e}}}{\text { Zhongwen } \mathrm{L}^{2, \mathrm{c}}}$, Chengcheng Guo ${ }^{1, \mathrm{~d}}$, and Yinbo

${ }^{1}$ Electronic Information School, WuHan University, WuHan 430072, China

${ }^{2}$ School of Information Science and Technology, Chengdu University, Chengdu 610106, China

aeisxujun@whu.edu.cn, ${ }^{*}$ yjf@whu.edu.cn (corresponding

author), ${ }^{c}$ netccg@whu.edu.cn, ${ }^{d}$ lizwxmu@gmail.com, ${ }^{\mathrm{e}} \mathrm{xyb} @$ whu.edu.cn

Keywords: packet scheduling, unreliable links, EDF.

\begin{abstract}
Unlike wired networks, links in wireless networks are unstable. Thus, considering the unstable links is important to design an efficient packet scheduling algorithm. We propose the Dividing Loss-aware Earliest Deadline First (DL-EDF) packet scheduling algorithm. In DL-EDF packet scheduling algorithm, the deadline of a packet is divided among the routing links and it is loss aware. The DL-EDF packet scheduling algorithm is compared with the frequently used EDF scheduling algorithm to demonstrate its efficiency.
\end{abstract}

\section{Introduction}

Wireless networks are widely adopted due to the development of wireless technologies and the flexibility of constructing. However, the wireless links are not so stable as the wired links because of the signal fading, interference, etc.. Hence, it's a challenge to gurantee the low packet loss rate and small delay in wireless networks.

The packet scheduling problems are studied in $[1,2,3,4]$. However, the unstable wireless links are not considered. In paper [5,6,7,8,9,10], the unstable wireless links are considered. In these studies, each user has the real-time throughput requirement which means the number of packets arrive at the destination nodes within a specific deadline should be greater than some bounds. The topology studied is star topology which is different from the general topology studied in this paper.

Considering that real-time requirements are widely proposed in industry scenarios, such as industry control. Thus, we study the real-time traffic. And time is divided into small slot. In the packet scheduling problem, we try to seek an efficient way to allocate time slots to packets in order to achieve high packet delivery ratio.

Unlike the previous studies, the packet deadlines and the average packet loss rates on links are jointly considered in the proposed DL-EDF (Dividing Loss-aware Earliest Deadline First) packet scheduling algorithm. The details of the DL-EDF algorithm will be introduce in section 3.

\section{Network Models}

Let $G=(V, E)$ represent the wireless mesh network, where $V$ is the node set, $E$ is the link set including the wireless connections between nodes. The packet set is represented by $F=\{f 1, f 2, \ldots \ldots$, $\mathrm{fN}$ \}. In total, there are $\mathrm{N}$ flows. In our model, each flow has a determined path from the source node si to the destination node di. We use $P_{i}=\left\{e_{i}^{1}, e_{i}^{2}, \ldots \ldots, e_{i}^{h_{i}}\right\}$ to represent the routing path of flow fi, and hi is the hop count of path $\mathrm{Pi}, e_{i}^{j}$ is the $\mathrm{j}$-th link in path $\mathrm{Pi}$. For each flow fi, we assume that its routing path $\mathrm{Pi}$ is generated by a certain routing protocol, such as the AODV, DSR, or OLSR routing protocols. Then all the packets in flow fi will follow the path Pi. If link e belongs to path $\mathrm{Pi}$, we set $\mathrm{xi}, \mathrm{e}=1$, otherwise $\mathrm{xi}, \mathrm{e}=0$.

All the packets in flow fi $(\mathrm{i}=1,2, \ldots, \mathrm{N})$ are expected to arrive at the destination node within the relative deadline Di. 


\subsection{Interference Model}

Three wireless interference models are widely adopted by researchers, which are primary interference model [11], protocol interference model [12] and physical interference model [13]. The primary interference model is too simply to capture the wireless channel characteristics. However, the physical interference model is too complex, thus it is hard to implement effective packet scheduling algorithms when adopting this model. Hence, we adopt the protocol interference model in this study. An example of the 2-hop interference model is introduced in Fig.1.

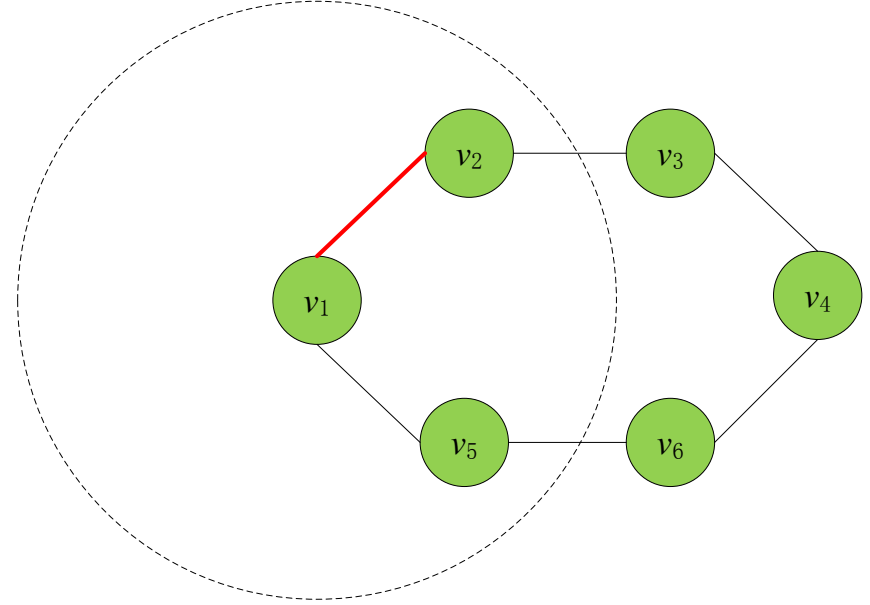

Fig.1 An example of the 2-hop interference model

In Fig.1, the dashed circle represents the interference range of node v1. When node v1 is transmitting packets to node v2 at slot t, then links (v1, v5), (v2, v3) and (v5, v6) can not transmit packets at slot $t$ because they interference with link (v1, v2). However, links (v3, v4) and (v4, v6) do not interference with link (v1, v2), thus one of them can transmit packets at slot $t$.

\subsection{Problem Formulation}

The problem considered in this paper aims to maximize the number of packets arriving at the destinations within the corresponding deadlines. And in each slot, the packets selected to be transmitted should not interference with each other.

Let assume that si,t equal to 1 when packet $\mathrm{i}$ is scheduled at slot $\mathrm{t}$, otherwise it is 0 . Let di represent the end-to-end delay of packet $\mathrm{i}$, and St denote the packets scheduled at slot $\mathrm{t}$. Let $\mathrm{I}(\mathrm{x})$ be an indication function, the value of $\mathrm{I}(\mathrm{x})$ is 1 when $\mathrm{x}$ is true, otherwise, it's 0 .

The mathmatical formulation of the packet scheduling problem can be described as follows.

$$
\max \cdot \sum_{i=1}^{N} I\left(d_{i}<D_{i}\right)
$$

s.t. the packets in $S_{t}$ do not interference with each other.

\section{DL-EDF packet scheduling algorithm}

\subsection{Calculate the weight of each packet.}

In this subsection, we introduce the key concepts when conducting the DL-EDF packet scheduling algorithm.

- $\quad$ Packet deadline. For packet $i$, let $D_{i}$ represent its deadline. Thus, packet $i$ should arrive at the destination node within $D_{i}$ slots. Otherwise, the packet is regarded as useless.

- The expected number of slots on a link. When packet $i$ requests to transmit on link $e$, and the reliability of link $e$ is $r_{e}$, then the expected number of slots required on link $e$ is $n_{e}=\frac{1}{r_{e}}$. 
- $\quad$ The expected number of slots on a path. For packet $i$, the expected number of total slots on its path is $n_{i}=\sum_{e \in P_{i}} \frac{1}{r_{e}}$.

- The weight of packet $i$ on link $e$. The weight of packet $i$ on link $e$ is defined as $w_{i, e}=D_{i} \times \frac{n_{e}}{n_{i}}$.

In the DL-EDF packet scheduling algorithm, the priorities of packets are assigned according to the weights of packets. The greater the weight is, the higher the priority is.

\subsection{Description of the algorithm.}

The pseudocode of the DL-EDF packet scheduling algorithm is shown in Table 1. Let assume that there are $\mathrm{N}$ packets to be scheduled in total, and the unscheduled packets are recorded in set $\mathrm{P}$.

Table 1. The pseudocode of the DL-EDF algorithm

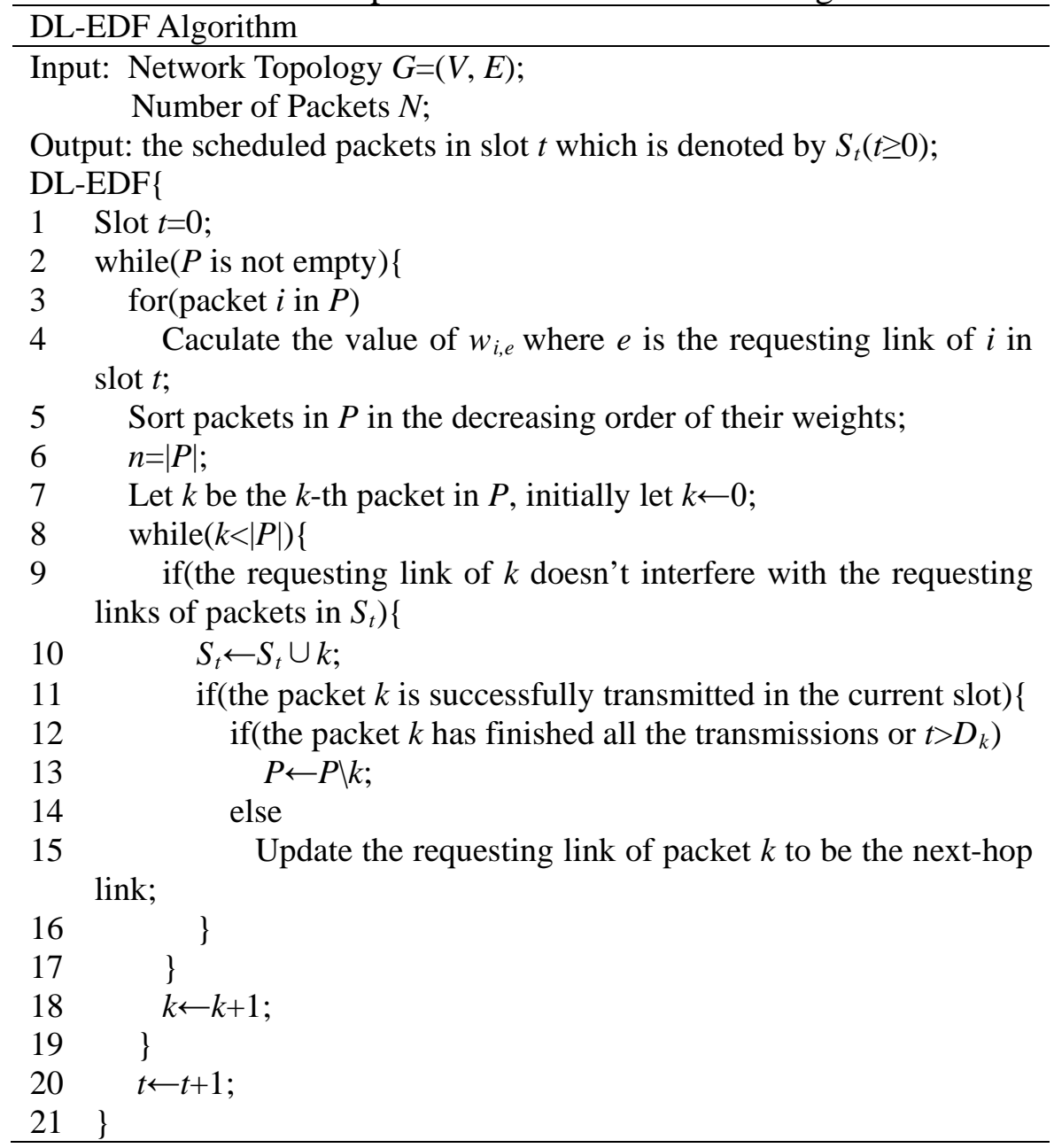

In each slot, we need to calculate the weights of all the $\mathrm{N}$ packets (line 3 to line 4 ) considering that the requesting links of a packet maybe different in different slots. Also, we need to visit all the packets in $\mathrm{P}$ in each slot, and select the ones which do not interfere with each other and have the highest weights (line 8 to line 19). When all the packets in set $\mathrm{P}$ are scheduled (missing deadline or arriving at the destination within deadline), the algorithm terminate (line 2).

\subsection{The time complexity of the algorithm.}

First, we analyze the time complexity of the packet scheduling in a single time slot. And then we analyze the time complexity of the DL-EDF packet scheduling algorithm.

Theorem 1. The time complexity of the single slot packet scheduling of the DL-EDF algorithm is $O\left(N n+N \log N+N^{2}\right)$. 
Proof. In the DL-EDF algorithm, it needs to update the weights of packets in each slot (line 3 to line 4). For any packet, calculating its weight needs to visit all the links in its path. Considering that the number of links in a path is no greater than the total number of links in the network, thus, the time complexity of line 3 to line 4 is is $O(N n)$. The time complexity of sorting the packets (line 5) can be accomplished within time steps $O(N \log N)$. The while iteration (line 8 to line 19) needs time step $O\left(N^{2}\right)$. Thus, the time complexity of the packet scheduling in a single slot is $O\left(N n+N \log N+N^{2}\right)$.

Theorem 2. The time complexity of the DL-EDF packet scheduling algorithm is $O\left(\left(N n+N \log N+N^{2}\right) \sum_{i=1}^{N} D_{i}\right)$.

Proof. Due to the unpredictable characteristic of the packet loss on wireless links, it's hard to estimate the actually number of slots needed to transmit a packet from the source node to the destination node. However, the number of slots will not exceed the deadline. Thus, in the worst case situation, the number of slots consumed by the $N$ packets will be $\sum_{i=1}^{N} D_{i}$. When all the links are in the same conflict area, that is, only one packet can be transmitted in a single slot, completing the transmission of the $N$ packets will need at most $\sum_{i=1}^{N} D_{i}$ slots. Theorem 1 has given the time complexity of packet scheduling in a single slot, thus the time complexity of the DL-EDF packet scheduling algorithm is $O\left(\left(N n+N \log N+N^{2}\right) \sum_{i=1}^{N} D_{i}\right)$.

\section{Simulation Results}

In this section, we compare the DL-EDF packet scheduling algorithm with the EDF scheduling algorithm. The routing algorithm adopted is the Dijkstra's algorithm. Thus, each packet will follow the shortest hop path from the source node to the destination node. The simulation topology is a $7 \times 7$ grid network which composes of 49 nodes and 84 links. The source and destination nodes of each packet are selected randomly. However, the source node and destination node of a packet should not be the same.

Note that the packet deadline is set to be $r n$, where $r$ is a random value and $n$ is the number of nodes in the network. In the simulation, we vary the number of packets from 200 to 1000 with an increment of 100 . The average packet loss rate on a link is in the range [0,0.4). The packet delivery ratios under the DL-EDF and EDF scheduling algorithms are shown in Fig.1. In Fig.1 (a), the random value $r$ is in the range of [0.5 2), while in Fig.1 (b), the value of $r$ is in the range of [0.5 3). From the two figures, we can see that the packet delivery ratios of the DL-EDF packet scheduling algorithm are higher than those of the EDF scheduling algorithm.

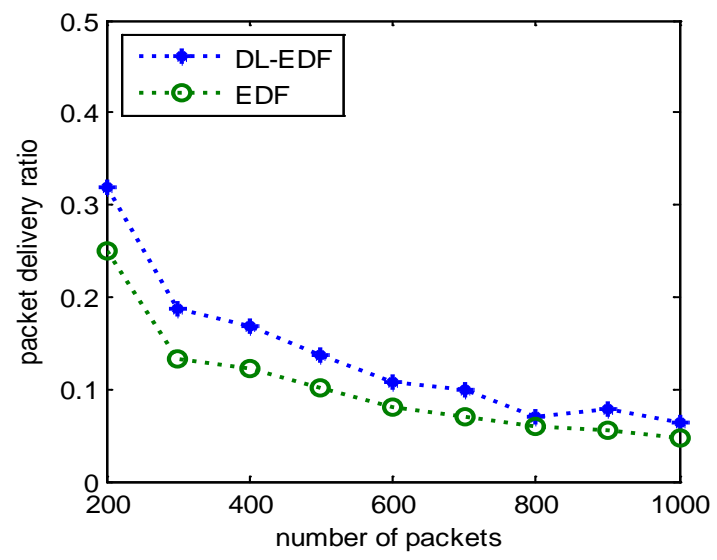

(a) $7 \times 7$ grid network, $r$ is in range of [0.5 2)

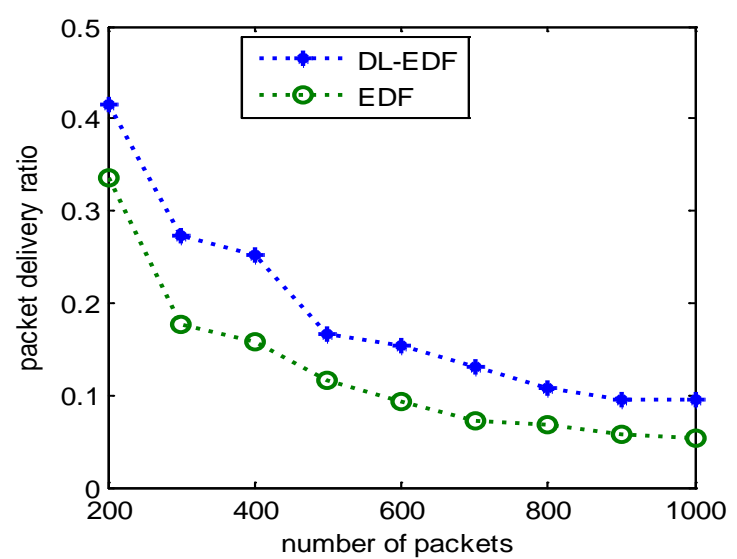

(b) $7 \times 7$ grid network, $r$ is in range of [0.5 3)

Fig.1. simulation results 


\section{Conclusions}

The DL-EDF packet scheduling algorithm is proposed. In this algorithm, the deadline of each packet is divided among the edges. And the average loss rate on each link is considered. The DLEDF packet scheduling algorithm is a polynomial time algorithm. With respect to the packet delivery ratios, the DL-EDF packet scheduling algorithm outperforms the EDF scheduling algorithm under the simulation settings.

\section{Acknowledgements}

This work was financially supported by the National High-tech R\&D Program of China (863 Program) (Grant No. 2012AA010904), the Scientific Research Fund of Sichuan Province, China (Grant No. 2014SZ0107), the Scientific Research Fund of Liaoning Province, China (No. L2013433), and the universities cooperation research project (20804).

\section{References}

[1]. Saifullah, A., et al. Real-Time Scheduling for WirelessHART Networks. in Real-Time Systems Symposium (RTSS), 2010 IEEE 31st. 2010.

[2]. Jun, Z. and Z. Dongmei. Connection-Based Scheduling for Supporting Real-Time Traffic in Wireless Mesh Networks. in Global Telecommunications Conference, 2008. IEEE GLOBECOM 2008. IEEE. 2008.

[3]. Wu, D., et al., Joint multi-radio multi-channel assignment, scheduling, and routing in wireless mesh networks. Wireless Networks, 2014. 20(1): p. 11-24.

[4]. Andrews, M. and L. Zhang. Minimizing end-to-end delay in high-speed networks with a simple coordinated schedule. in INFOCOM'99. Eighteenth Annual Joint Conference of the IEEE Computer and Communications Societies. Proceedings. IEEE. 1999: IEEE.

[5]. Shakkottai, S. and R. Srikant, Scheduling Real-Time Traffic With Deadlines over a Wireless Channel. Wireless Networks, 2002. 8(1): p. 13-26.

[6]. Hou, I.H. and P. Kumar, A survey of recent results on real-time wireless networking. Proceedings of Real-Time Wireless for Industrial Applications, 2011.

[7]. I-Hong, H., V. Borkar and P.R. Kumar. A Theory of QoS for Wireless. in INFOCOM 2009. 2009.

[8]. Hou, I.H. and P. Kumar, Admission control and scheduling for QoS guarantees for variablebit-rate applications on wireless channels. MobiHoc'09, 2009: p. 175-184.

[9]. I-Hong, H. and P.R. Kumar. Scheduling Heterogeneous Real-Time Traffic over Fading Wireless Channels. in INFOCOM, 2010 Proceedings IEEE. 2010.

[10]. Hou, I. Providing end-to-end delay guarantees for multi-hop wireless sensor networks. in Global Communications Conference (GLOBECOM), 2013 IEEE. 2013.

[11]. Yi, S., et al., Bridging the Gap between Protocol and Physical Models for Wireless Networks. Mobile Computing, IEEE Transactions on, 2013. 12(7): p. 1404-1416.

[12]. Shi, Y., et al. How to correctly use the protocol interference model for multi-hop wireless networks. in Proceedings of the tenth ACM international symposium on Mobile ad hoc networking and computing. 2009: ACM.

[13]. Gupta, P. and P.R. Kumar, The capacity of wireless networks. Information Theory, IEEE Transactions on, 2000. 46(2): p. 388-404. 